\title{
Government transparency measurement through prioritized distance operators
}

\author{
Ezequiel Avilés-Ochoa ${ }^{\mathrm{a}, *}$, Ernesto León-Castro ${ }^{\mathrm{a}}$, Luis Alessandri Perez-Arellano ${ }^{\mathrm{a}}$ \\ and José M. Merigób \\ ${ }^{a}$ University of Occidente, Blvd. Lola Beltrán s/n esq. Circuito Vial, Culiacán, México \\ ${ }^{\mathrm{b}}$ Department of Management Control and Information Systems, School of Economics and Business, \\ University of Chile, Av. Diagonal Paraguay, Santiago, Chile
}

\begin{abstract}
The prioritized induced probabilistic ordered weighted average distance (PIPOWAD) has been developed. This new operator is an extension of the ordered weighted average (OWA) operator that can be used in cases where we have two sets of data that want to be compared. Some of the main characteristics of this new operator are: 1) Not all the decision makers are equally important, so the information needs to be prioritized, 2) The information has a probability to occur and 3) The decision makers can change the importance of the information based in an induced variable. Additionally, characteristics and families of the PIPOWAD operator are presented. Finally, an application of the PIPOWAD operator in order to measure government transparency in Mexico is presented.
\end{abstract}

Keywords: OWA operator, prioritized aggregation operators, induced aggregation operators, probabilistic aggregation operators, transparency and access to information

\section{Introduction}

Transparency and access to information to citizens has been one of the important aspects for democratic countries, the two elements that are needed to achieve this are access and communication [4]. The objective to do this, is to know how the government is making decisions to prevent corruption, request information and be able to bring this to all the people $[3,17,18]$. Among the methodologies that are used in Mexico to measure government transparency is the Index of the Right of Access to Information in Mexico (IDAIM is the acronym in Spanish) is an index that measures the level of transparency of states. One of the advantages of this index is that it is possible to compare the states with higher transparency and detect which are the items that the other states should work to achieve

\footnotetext{
* Corresponding author. Ezequiel Avilés-Ochoa, University of Occidente, Blvd. Lola Beltrán s/n esq. Circuito Vial, Culiacán 80200, México. E-mail: ezequiel.aviles@udo.mx.
}

that level. Some decision-making methods [9, 37, 47] are based on distance measures [16], with the Hamming distance [45] being one of the most common distance methods that compares two variables, helping the decision maker to understand the difference between the ideal situation and the real one.

Among the combinations that have been made with distance techniques are the Normalized Hamming Distance (NHD) that include arithmetic mean in the formulation or the Weighted Hamming distance (WHD) when weighted average is included. One combination that has been used in this paper is based on the Ordered Weighted Average (OWA) operator [42] to obtain the OWA Distance (OWAD) operator [22, 46, 52, 59]. Among the extension that have developed are the one using induced operators $[23,24]$, intuitionistic fuzzy sets [49] and Bonferroni means [10].

The aim of this paper is to present some new aggregation operators based on the Prioritized Induced 
Probabilistic Ordered Weighted Average Distance (PIPOWAD) operator. It is a new aggregation operator that introduces the Hamming distance into the PIPOWA operator [34]. The primary advantage of this new operator is that one formulation combines four different operators: 1) the Prioritized OWA (PrOWA) operator [39], that has developed different extension such as intuitionistic fuzzy prioritized OWA operator [37], hesitant fuzzy prioritized operators [5] and so on. Also, many applications in decision making problems and multiple attribute decision making has also been developed [32, 48], 2) the Probabilistic OWA (POWA) operator [30], that some extension using interval numbers [50] and linguistic variables [28]. In addition, some applications in finance and fuzzy environment have been developed $[35,52], 3)$ the Induced OWA (IOWA) operator [38], that has developed extensions using moving average, heavy weights and intuitionistic fuzzy sets $[6,51]$ and some applications in multiple criteria decision making, safety programs and cluster analysis have been done $[5,11,56]$ and 4) the Hamming distance operator [45] that has been applied in many areas such as sports and finance $[8,19]$.

A generalization of the PIPOWAD operator is presented by using quasi-arithmetic means [21, 23]. In this manner, we obtained the Quasi-PIPOWAD operator. The advantage of using the quasi-arithmetic means is that they includes a wide range of aggregation operators, such as a generalized operator, quadratic aggregation, geometric aggregation, maximum operator, minimum operator and others.

Additionally, some specific cases of the PIPOWAD operator are presented as families. These cases are important because they can be used when the problem is simple, and all the elements and information needed to use the PIPOWAD operator are not necessary. Among the specific cases are the probabilistic maximum, the probabilistic minimum, the PIOWAD operator, and the PPOWAD operator.

This new operator was used in order to measure government transparency based on the IDAIM index in Mexico. The information was obtained by three different experts that have been working in governmental dependence in the field and based on their knowledge and expectations the calculation for Durango has been made using different operators. It can be seen, that if we take different information it is possible to compare different scenarios that will give the decision makers an opportunity to know in what they can work in order to achieve better results in future years.
The paper is organized as follows: In Section 2, we review some aggregation operators. Section 3 introduces the PIPOWAD operator, and Section 4 develops the generalized PIPOWAD operator. Section 5 explains the steps for the use of this operator in financial selection, and Section 6 presents the use of the PIPOWAD operator in a financial selection case. Section 7 summarizes the primary conclusions of the paper.

\section{Preliminaries}

\subsection{OWA operator and main extensions}

The OWA operator introduced by Yager [42] is an aggregation operator that provides a parameterized family of aggregation operators between the minimum and the maximum. It can be defined as follows:

Definition 1. An OWA operator of dimension $n$ is a mapping of $O W A: R^{n} \rightarrow R$ with a weight vector $\mathrm{W}$ of dimension $\mathrm{n}$ with $\sum_{i=1}^{n} w_{i}=1$ and $w_{i} \in[0,1]$, such that:

$$
O W A\left(a_{1}, a_{2}, \ldots, a_{n}\right)=\sum_{j=1}^{n} w_{j} b_{j}
$$

where $b_{j}$ is the jth element and the largest of the collection $a_{1}, a_{2}, \ldots, a_{n}$.

The prioritized OWA (PrOWA) operator developed by Yager [41] is an aggregation operator that is useful when problem-solving decision makers do not have the same standing in the final decision. Thus, this operator allocates additional impact to some decision makers and less to others. This operator is formulated with the following expression $[14,43,44]$ :

Definition 2. Assume that a collection of criteria is divided into q distinct groups, $H_{1}, H_{2}, \ldots, H_{q}$, for which $H_{i}=\left\{C_{i 1}, C_{i 2}, \ldots, C_{i n}\right\}$ denotes the criteria of the $i$ th category $(i=1, \ldots, q)$ and $n_{i}$ is the number of criteria in the class. Furthermore, we have a prioritization between the groups so that $H_{1}>$ $H_{2}>\ldots>H_{q}$. That is, the criteria in the category $H_{i}$ have a higher priority than those in $H_{k}$ for all $i<k$ and $i, k \in\{1, \ldots, q\}$. We denote the total set of criteria as $C=U_{i=1}^{q} H_{i}$ and the total number of criteria as $n=\sum_{i=1}^{q} n_{i}$. Additionally, suppose $X=$ $\left\{x_{1}, \ldots, x_{m}\right\}$ indicates the set of alternatives. For a given alternative x, let $C_{i j}(x)$ measure the satisfaction of the $j$ th criteria in the $i$ th group by alternative $x \in X$, 
for each $i=1, \ldots, q, j=1, \ldots, i_{i}$. The formula is as follows:

$$
C_{(x)}=\sum_{i=1}^{q} \sum_{h=1}^{n_{i}} w_{i j} C_{i j}(x)
$$

where $w_{i j}$ is the corresponding weight of the $j$ th criteria in the $i$ th category and $i=1, \ldots, q$ and $j=$ $1, \ldots, i_{i}$. If $w_{i}=1 / n$ for all $\mathrm{i}$, the PrOWA becomes the prioritized average (PrA).

Another extension of the OWA used in this paper is the probabilistic OWA (POWA) operator. This operator uses a weighted vector and a probability vector, making it possible to underestimate or overestimate based on the knowledge and attitude of the decision maker [30]. Another extension that is used in this paper is the induced OWA (IOWA) operator [38]. The main characteristic of this operator is that the weights are not assigned based on the value of the argument, but instead they are induced based on the knowledge or expectations of the decision maker. Along with the POWA operator, the PrOWA operator and the IOWA operator, several new extensions have been developed. One of them is the induced probabilistic (IPOWA) operator, which includes ordering the weights based on the induced values and a second probability vector in the same formulation [27].

The prioritized induced probabilistic OWA (PIPOWA) operator is an operator that considers the primary characteristics of the POWA, PrOWA and IOWA operators. This operator is useful in group decision-making problems, where it considers prioritized criteria among the decision makers, a probability vector and an induced-ordered weighting vector. The definition is as follows [34]:

Definition 3. A prioritized induced probabilistic OWA (PIPOWA) operator of dimension $\mathrm{n}$ is a mapping of IPOWA : $R^{n} x R^{n} \rightarrow R$ that has an associated weight vector $\mathrm{w}$ of dimension $n$, where $w_{j} \in[0,1]$ and $\sum_{j=1}^{n} w_{j}=1$, so that

$$
\begin{aligned}
& \text { PIPOWA }\left(u_{1}, a_{1}, u_{2}, a_{2}, \ldots, u_{n}, a_{n}\right) \\
& =\sum_{i=1}^{q} \sum_{h=1}^{n_{i}} b_{j} \hat{v}_{i j} C_{i j}(x)
\end{aligned}
$$

where $b_{j}$ is the jth element that has the largest value of $u_{t} . u_{t}$ is the induced order of the variables, $\hat{v}_{i j}$ is the corresponding weight of the $j$ th criteria in the $i$ th category, $i=1, \ldots, q, j=1, \ldots, i_{i}$, and $C_{i j}(x)$ measures the satisfaction of the $j$ th criteria in the $i$ th group by alternative $x \in X$, for each $i=$
$1, \ldots, q, j=1, \ldots, i_{i}$. Additionally, each element has an associated probability $p_{i}$ with $\sum_{i=1}^{n} p_{i}=1$ and $p_{i} \in[0,1], \hat{v}_{j}=\beta w_{j}+(1-\beta) p_{j}$, where $\beta \in$ $[0,1]$ and $p_{j}$ is the probability of $p_{i}$.

\subsection{Distance techniques}

Distance techniques are methodologies that can compare two set of elements to determine the distance between them, allowing selection of the alternative that is closer to the ideal set of data. The Hamming distance [45] is a classical tool that can be used with fuzzy sets, interval-valued fuzzy sets, intuitionistic fuzzy sets and Bonferroni means [23, 26, 49, 57]. Some of the basic properties of distance techniques are [25] as follows:

a) Non-negativity: $D\left(A_{1}, A_{2}\right) \geq 0$;

b) Commutativity: $D\left(A_{1}, A_{2}\right)=D\left(A_{2}, A_{1}\right)$;

c) Reflexivity: $D\left(A_{1}, A_{2}\right)=0$; and

d) Triangle inequality: $D\left(A_{1}, A_{2}\right)+D\left(A_{2}, A_{3}\right)$ $\geq D\left(A_{1}, A_{3}\right)$.

The Hamming distance can be defined as follows [28] when combining it with the OWA operator [20, 46, 59]:

Definition 4. An OWAD operator of dimension $\mathrm{n}$ is a mapping of $O W A D:[0,1]^{n} x[0,1]^{n} \rightarrow[0,1]$ that has an associated weighting vector $w$, with $\sum_{j=1}^{n} w_{j}=1$ and $w_{j} \in[0,1]$ such that

$$
O W A D\left(\left\langle x_{1}, y_{1}\right\rangle, \ldots,\left\langle x_{n}, y_{n}\right\rangle\right)=\sum_{j=1}^{n} w_{j} D_{j}
$$

where $D_{j}$ is the jth largest of the differences $\left|x_{i}-y_{i}\right|$ and $\left|x_{i}-y_{i}\right|$ is the argument variable represented in the form of individual distances.

The probabilistic OWA distance (POWAD) operator is another extension that uses the distance measure, probabilities and OWA operator in the same formulation and the induced OWA distance [31] (IOWAD) operator by including an induced reordering step [23].

Additionally, distance measures can be added in the prioritized OWA operator, obtaining the prioritized OWA distance (PrOWAD) operator, which is defined as follows:

Definition 5. Assume a collection of criteria portioned into q distinct groups, $H_{1}, H_{2}, \ldots, H_{q}$ for which $H_{i}=\left\{C_{i 1}, C_{i 2}, \ldots, C_{i n}\right\}$ denotes the criteria of the $i$ th category $(i=1, \ldots, q)$ and $n_{i}$ is the 
number of criteria in the class. Furthermore, we have a prioritization between the groups such that $H_{1}>H_{2}>\ldots>H_{q}$. That is, the criteria in category $H_{i}$ have a higher priority than those in $H_{k}$ for all $i<k$ and $i, k \in\{1, \ldots, q\}$. We denote the total set of criteria as $C=U_{i=1}^{q} H_{i}$ and the total number of criteria as $n=\sum_{i=1}^{q} n_{i}$. Additionally, suppose $X=\left\{x_{1}, \ldots, x_{m}\right\}$ indicates the set of alternatives and $Y=\left\{y_{1}, \ldots, y_{m}\right\}$ indicates the ideal value of the alternatives. For a given set of data $\mathrm{z}$ that is defined by $\left|x_{i}-y_{i}\right|$, let $C_{i j}(z)$ measure the satisfaction of the $j$ th criteria in the $i$ th group, for each $i=1, \ldots, q, j=$ $1, \ldots, i_{i}$. The formula is as follows:

$$
\operatorname{PrOWAD}\left(C_{\left(x_{n}, y_{n}\right)}\right)=\sum_{i=1}^{q} \sum_{h=1}^{n_{i}} w_{i j} C_{i j}(z)
$$

where $C_{i j}(z)$ is the $\left|x_{i}-y_{i}\right|$ value of each criteria and $w_{i j}$ is the corresponding weight of the $j$ th criteria in the $i$ th category, $i=1, \ldots, q, j=1, \ldots, i_{i}$.

\section{The PIPOWAD operator}

\subsection{Theoretical foundations}

The prioritized induced probabilistic ordered weighted average distance (PIPOWAD) operator is an aggregation operator that includes in the same formulation probabilities, induced variables, prioritized variables and distance techniques. This new operator can be used for different types of problems and generates additional, new scenarios. It is important to note that the PIPOWAD operator includes specific cases, such as the prioritized probabilistic ordered weighted average distance (PPOWAD) operator and the prioritized induced ordered weighted average distance (PIOWAD) operator. The PIPOWAD operator is defined as follows:

Definition 6. A prioritized induced probabilistic OWA distance (PIPOWAD) operator of dimension $\mathrm{n}$ is a mapping of PIPOWAD : $R^{n} x R^{n} \rightarrow R$ that has an associated weight vector $\mathrm{w}$ of dimension $n$ where $w_{j} \in[0,1]$ and $\sum_{j=1}^{n} w_{j}=1$, so that

$$
\begin{aligned}
& \text { PIPOWAD }\left(u_{1}, x_{1}, y_{1}, \ldots, u_{n}, x_{n}, y_{n}\right) \\
& =\sum_{i=1}^{q} \sum_{h=1}^{n_{i}} b_{j} \hat{v}_{i j} C_{i j}(z)
\end{aligned}
$$

where $b_{j}$ is the jth largest of the differences $\left|x_{i}-y_{i}\right|$, $\left|x_{i}-y_{i}\right|$ is the argument variable represented in the form of individual distances based on $u_{t}, u_{t}$ is the induced order of variables, $\hat{v}_{i j}$ is the corresponding weight of the $j$ th criteria in the $i$ th category, $i=1, \ldots, q, j=1, \ldots, i_{i}$, and $C_{i j}(z)$ measures the satisfaction of the $j$ th criteria in the $i$ th group by the $\left|x_{i}-y_{i}\right|$ value of each criteria, for each $i=$ $1, \ldots, q, j=1, \ldots, i_{i}$. Additionally, each element has an associated probability $p_{i}$ with $\sum_{i=1}^{n} p_{i}=1$ and $p_{i} \in[0,1], \hat{v}_{j}=\beta w_{j}+(1-\beta) p_{j}$, where $\beta \in$ $[0,1]$ and $p_{j}$ is the probability of $p_{i}$.

In the case where the weights in the problem are not induced, the PIPOWAD operator becomes the PPOWAD operator. The definition is as follows:

Definition 7. A prioritized probabilistic OWA distance (PPOWAD) operator of dimension $\mathrm{n}$ is a mapping of PPOWAD : $R^{n} x R^{n} \rightarrow R$ that has an associated weight vector $\mathrm{W}$ of dimension $n$, where $w_{j} \in[0,1]$ and $\sum_{j=1}^{n} w_{j}=1$, so that

$$
\begin{aligned}
& \text { PPOWAD }\left(\left\langle x_{1}, y_{1}\right\rangle,\left\langle x_{2}, y_{2}\right\rangle, \ldots,\left\langle x_{n}, y_{n}\right\rangle\right) \\
& =\sum_{i=1}^{q} \sum_{h=1}^{n_{i}} b_{j} \hat{v}_{i j} C_{i j}(z)
\end{aligned}
$$

where $b_{j}$ is the jth largest of the differences $\left|x_{i}-y_{i}\right|$, $\hat{v}_{i j}$ is the corresponding weight of the $j$ th criteria in the $i$ th category, $i=1, \ldots, q, j=1, \ldots, i_{i}$, and $C_{i j}(z)$ measures the satisfaction of the $j$ th criteria in the $i$ th group by the $\left|x_{i}-y_{i}\right|$ value of each criteria, for each $i=1, \ldots, q, j=1, \ldots, i_{i}$. Additionally, each element has an associated probability $p_{i}$ with $\sum_{i=1}^{n} p_{i}=1$ and $p_{i} \in[0,1], \hat{v}_{j}=\beta w_{j}+$ $(1-\beta) p_{j}$, where $\beta \in[0,1]$ and $p_{j}$ is the probability of $p_{i}$.

Another specific case is when there is no probability vector in the formulation, so the PIPOWAD operator becomes the PIOWAD operator. The definition is as follows:

Definition 8. A prioritized induced OWA distance (PIOWAD) operator of dimension $\mathrm{n}$ is a mapping of PIOWAD : $R^{n} x R^{n} \rightarrow R$ that has an associated weight vector $\mathrm{w}$ of dimension $n$, where $w_{j} \in[0,1]$ and $\sum_{j=1}^{n} w_{j}=1$, so that

$$
\begin{aligned}
& \text { PIOWAD }\left(\left\langle u_{1}, x_{1}, y_{1}\right\rangle, \ldots,\left\langle u_{n}, x_{n}, y_{n}\right\rangle\right) \\
& =\sum_{i=1}^{q} \sum_{h=1}^{n_{i}} b_{j} \hat{v}_{i j} C_{i j}(x)
\end{aligned}
$$

where $b_{j}$ is the jth largest of the differences $\left|x_{i}-y_{i}\right|$, $\left|x_{i}-y_{i}\right|$ is the argument variable represented in the form of individual distances based on $u_{t}, u_{i}$ is the 
Table 1

Information provide by expert 1

\begin{tabular}{cccc}
\hline & $c_{1}$ & $c_{2}$ & $c_{3}$ \\
\hline$a_{1}$ & 5 & 7 & 6 \\
$a_{2}$ & 4 & 6 & 8 \\
$a_{3}$ & 4 & 5 & 5 \\
\hline
\end{tabular}

Table 2

Information provide by expert 2

\begin{tabular}{lccc}
\hline & $c_{1}$ & $c_{2}$ & $c_{3}$ \\
\hline$a_{1}$ & 3 & 6 & 7 \\
$a_{2}$ & 6 & 7 & 6 \\
$a_{3}$ & 5 & 5 & 5 \\
\hline
\end{tabular}

Table 3

Information provide by expert 3

\begin{tabular}{cccc}
\hline & $c_{1}$ & $c_{2}$ & $c_{3}$ \\
\hline$a_{1}$ & 6 & 6 & 7 \\
$a_{2}$ & 7 & 4 & 7 \\
$a_{3}$ & 5 & 6 & 6 \\
\hline
\end{tabular}

Table 4

Best scenario

\begin{tabular}{cccc}
\hline & $c_{1}$ & $c_{2}$ & $c_{3}$ \\
\hline$b_{1}$ & 8 & 9 & 9 \\
\hline
\end{tabular}

induced order of the variables, $\hat{v}_{i j}$ is the corresponding weight of the $j$ th criteria in the $i$ th category, $i=1, \ldots, q, j=1, \ldots, i_{i}$ and $C_{i j}(x)$ measures the satisfaction of the $j$ th criteria in the $i$ th group by alternative $x \in X$, for each $i=1, \ldots, q, j=1, \ldots, i_{i}$.

To obtain a clear vision of the process to generate the results of the PIPOWAD operator, we present the following example:

Example 1. Assume a problem using three different experts in the decision-making process $\left(e_{1}, e_{2}, e_{3}\right)$, who want to evaluate three different scenarios $\left(a_{1}, a_{2}, a_{3}\right)$ with three different components $\left(c_{1}, c_{2}, c_{3}\right)$ in comparison to the optimum scenario $b_{1}$. Additionally, the following vectors are used: a weighting vector $\mathrm{W}(W=0.30,0.25,0.45)$, an induced vector $\mathrm{U}(U=5,15,10)$, a probabilistic vector $\mathrm{P}(P=0,35,0.35,0.30)$ and a prioritized vector $\operatorname{Pr}(\operatorname{Pr}=0.20,0.35,0.45)$. The information is as follow (See Tables 1-4).

The result for $a_{1}$ is as follows:

$$
\begin{aligned}
c_{1}= & (0.20)(8-5)+(0.35)(8-3) \\
& +(0.45)(8-6)=0.35
\end{aligned}
$$

The same process is performed for $c_{2}$ and $c_{3}$, obtaining prioritized distance results $(3.25,2.8,2.2)$ that are then multiplied to produce the results for the induced weighting and probabilistic vectors, as follows:

$$
\begin{aligned}
& c_{1}=(3.25)(0.45)(0.35)=0.511 \\
& c_{2}=(2.8)(0.25)(0.30)=0.245 \\
& c_{3}=(2.2)(0.30)(0.45)=0.198
\end{aligned}
$$

Obtaining a result of $a_{1}=0.954$.

The same process is performed for $a_{2}$ and $a_{3}$. The results are $a_{2}=0.811$ and $a_{3}=1.134$. In this sense, the best scenario is for $a_{2}>a_{1}>a_{3}$.

\subsection{Families of the PIPOWAD operator}

An important aspect of the PIPOWAD operator is that it includes a series of different operators if the weighting vector $\mathrm{W}$ and the coefficient $\beta$ of the probability vector are analyzed. The main advantage of the families of the PIPOWAD operator is that if the problem is simple, it is possible to use other operators in the same family, such as the PPOWAD operator and the PIOWAD operator. Based on the coefficient $\beta$ and weighting vector $\mathrm{W}$, we obtain the following specific cases $[21,25]$, as follows:

a) Use the PPOWAD operator if $\beta=0$ and the PIOWAD operator if $\beta=1$. It is important to note that the closer $\beta$ is to 1 , the more importance is given to the PIOWAD operator, and vice versa;

b) The minimum distance when $w_{n}=1$ and $w_{j}=$ 0 , for all $j \neq n$ and $\beta=0$;

c) The PIPOWA operator is obtained if one of the sets is empty;

d) The probabilistic maximum ( $\mathrm{wp}=1$ and $w j=0$, for all $\mathrm{j} \neq \mathrm{p}$, and up $=\operatorname{Max}\{\mathrm{ai}\})$;

e) The probabilistic minimum ( $w p=1$ and $w j=0$, for all $\mathrm{j} \neq \mathrm{p}$, and $\mathrm{up}=\operatorname{Min}\{$ ai $\})$;

f) The arithmetic probabilistic approach ( $w j=1 / \mathrm{n}$, for all ai);

g) The step-PIPOWAD operator $(\mathrm{wk}=1$ and $w j=0$, for all $\mathrm{j} \neq \mathrm{k}$ );

h) The general olympic-PIPOWAD operator $(w j=0$ for $\mathrm{j}=1,2, \ldots, \mathrm{k}, \mathrm{n}, \mathrm{n}-1, \ldots, \mathrm{n}$ $-\mathrm{k}+1$; and for all others, $\mathrm{wj}^{*}=1 /(\mathrm{n}-2 \mathrm{k})$, where $\mathrm{k}<\mathrm{n} / 2$ ); and

i) The centered-PIPOWAD operator (if it is symmetric, strongly decaying from the center to the maximum and the minimum, and inclusive). 
Families of the HPIPOWAD operator

\begin{tabular}{l} 
Particular case \\
\hline$p_{i}=\frac{1}{n}$, for all $i$ \\
$w_{i}=\frac{1}{n}$, for all $I$ \\
$u_{i}=\frac{1}{n}$, for all $i$
\end{tabular}

When all decision makers are equally important

When there is not probabilistic, prioritized and induced vector

When there is only probabilistic vector

When only an order induced vector is used

When only a prioritized vector is used
Heavy prioritized induced weighted average distance (HPIOWAD) operator Heavy prioritized induced probabilistic average distance (HPIPAD) operator Heavy prioritized probabilistic weighted average distance (HPPWAD) operator Heavy induced probabilistic weighted average distance (HIPWAD) operator Heavy ordered weighted distance (HOWAD) operator

Heavy probabilistic ordered weighted average distance (HPOWAD) operator Heavy induced ordered weighted average distance (HIOWAD) operator Heavy prioritized ordered weighted average distance (HPrOWAD)

\subsection{Heavy aggregation operators in the PIPOWAD operator}

An interesting extension of the OWA operator is the heavy ordered weighted average (HOWA) operator (Yager, 2002). In this formulation the weighting vector is expanded, and it is not bounded to 1 . In this sense, the weighting vector can range from 1 to $\infty$ or even $-\infty$ to $\infty$. This operator has been expanded using order induced variables, moving average and other techniques [6]. The formulation is as follows.

Definition 9. A heavy aggregation operator, is an extension to OWA operator that allows the weight vector goes up to $n$. So a HOWA operator is an application $R^{n} \rightarrow R$ which are associated to a weight vector w which $w_{j} \in[0,1]$ and $1 \leq \sum_{j=1}^{n} w_{j} \leq n$, so that

$$
\text { HOWA }\left(a_{1}, a_{2}, \ldots, a_{n}\right)=\sum_{j=1}^{n} w_{j} b_{j}
$$

being $b_{j}$ the jth largest element of the collection $a_{1}, a_{2}, \ldots, a_{n}$.

So, if the weighting vector that is used in the PIPOWAD operator is unbounded, then the heavy prioritized induced probabilistic ordered weighted average distance (HPIPOWAD) operator is obtained. The formulation is as follows.

Definition 10. A heavy prioritized induced probabilistic OWA distance (HPIPOWAD) operator of dimension $\mathrm{n}$ is a mapping of HPIPOWAD : $R^{n} x R^{n} \rightarrow R$ that has an associated weight vector $\mathrm{w}$ of dimension $n$ where $w_{j} \in[0,1]$ and $1 \leq \sum_{j=1}^{n} w_{j}$ $\leq n$, so that

$$
\begin{aligned}
& \text { HPIPOWAD }\left(\left\langle u_{1}, x_{1}, y_{1}\right\rangle, \ldots,\left\langle u_{n}, x_{n}, y_{n}\right\rangle\right) \\
& =\sum_{i=1}^{q} \sum_{h=1}^{n_{i}} b_{j} \hat{v}_{i j} C_{i j}(z)
\end{aligned}
$$

where $b_{j}$ is the jth largest of the differences $\left|x_{i}-y_{i}\right|$, $\left|x_{i}-y_{i}\right|$ is the argument variable represented in the form of individual distances based on $u_{t}, u_{t}$ is the induced order of variables, $\hat{v}_{i j}$ is the corresponding weight of the $j$ th criteria in the $i$ th category, $i=1, \ldots, q, j=1, \ldots, i_{i}$, and $C_{i j}(z)$ measures the satisfaction of the $j$ th criteria in the $i$ th group by the $\left|x_{i}-y_{i}\right|$ value of each criteria, for each $i=$ $1, \ldots, q, j=1, \ldots, i_{i}$. Additionally, each element has an associated probability $p_{i}$ with $\sum_{i=1}^{n} p_{i}=1$ and $p_{i} \in[0,1], \hat{v}_{j}=\beta w_{j}+(1-\beta) p_{j}$, where $\beta \in$ $[0,1]$ and $p_{j}$ is the probability of $p_{i}$. It is important to note that in this formulation the weighting vector can also range from $-\infty$ to $\infty$.

Some of the families of the HPIPOWAD operator can be seen in Table 5 .

\section{Generalized aggregation operators with the PIPOWAD operator}

In this section, we present some generalizations based on the generalized OWA (GOWA) operator [2, 15, 39]. The generalized PIPOWAD (GPIPOWAD) includes a wide range of aggregation operators, including the quasi-arithmetic means (Quasi-PIPOWAD) operator. We focus on the latter because it includes the generalized means as a specific case. It is important to note that these new formulations are critical because they assist in solving more complex problems based on the information available and expectations, creating new decision-making scenarios. In the following, the definitions of the Quasi-PIPOWAD operator, the Quasi-PIOWAD operator and the Quasi-PPOWAD operator are presented:

Definition 11. A quasi-prioritized induced probabilistic OWA distance (Quasi-PIPOWAD) operator of dimension $\mathrm{n}$ is a mapping of Quasi-PPOWAD : $R^{n} x R^{n} \rightarrow R$ that has an associated weight vector $\mathrm{w}$ 
Table 6

Families of generalized PIPOWAD operators

\begin{tabular}{ll}
\hline Particular case & Quasi-PIPOWAD \\
\hline$p_{i}=\frac{1}{n}$, for all $i$ & Quasi-arithmetic prioritized induced weighted average distance (Quasi-PIOWAD) operator \\
$w_{i}=\frac{1}{n}$, for all $i$ & Quasi-arithmetic prioritized induced probabilistic average distance (Quasi-PIPAD) operator \\
$g(b)=b^{\lambda}$ & Generalized PIPOWAD (Minkowski) operator \\
$g(b)=b$ & PIPOWAD operator \\
$g(b)=b^{2}$ & Prioritized induced probabilistic ordered weighted quadratic average (PIPOWQAD) operator \\
$g(b) \rightarrow b^{\lambda}$, for $\lambda \rightarrow 0$ & Prioritized induced probabilistic ordered weighted geometric average (PIPOWGAD) operator \\
$g(b)=b^{-1}$ & Prioritized induced probabilistic ordered weighted harmonic average (PIPOWHAD) operator \\
$g(b)=b^{3}$ & Prioritized induced probabilistic ordered weighted cubic average (PIPOWCAD) operator \\
$g(b) \rightarrow b^{\lambda}$, for $\lambda \rightarrow \infty$ & Maximum operator \\
$g(b) \rightarrow b^{\lambda}$, for $\lambda \rightarrow-\infty$ & Minimum operator
\end{tabular}

of dimension $n$ where $w_{j} \in[0,1]$ and $\sum_{j=1}^{n} w_{j}=1$, so that

$$
\begin{aligned}
& \text { Quasi-PIPOWAD }\left(\left\langle u_{1}, x_{1}, y_{1}\right\rangle, \ldots,\left\langle u_{n}, x_{n}, y_{n}\right\rangle\right) \\
& =g^{-1} \sum_{i=1}^{q} \sum_{h=1}^{n_{i}} b_{j} \hat{v}_{i j} g\left(C_{i j}(z)\right)
\end{aligned}
$$

where $b_{j}$ is the jth largest of the differences $\left|x_{i}-y_{i}\right|$, $\left|x_{i}-y_{i}\right|$ is the argument variable represented in the form of individual distances according to $u_{t}, u_{t}$ is the induced order of the variables, $\hat{v}_{i j}$ is the corresponding weight of the $j$ th criteria in the $i$ th category, $i=1, \ldots, q, j=1, \ldots, i_{i}$, and $C_{i j}(z)$ measures the satisfaction of the $j$ th criteria in the $i$ th group by the $\left|x_{i}-y_{i}\right|$ value of each criteria, for each $i=$ $1, \ldots, q, j=1, \ldots, i_{i}$. Additionally, each element has an associated probability $p_{i}$ with $\sum_{i=1}^{n} p_{i}=1$ and $p_{i} \in[0,1], \hat{v}_{j}=\beta w_{j}+(1-\beta) p_{j}$, where $\beta \in$ $[0,1], p_{j}$ is the probability of $p_{i}$ and $g\left(C_{i j}(z)\right)$ is a continuous strictly monotonic function.

Definition 12. A quasi-prioritized probabilistic OWA distance (Quasi-PPOWAD) of dimension $\mathrm{n}$ is a mapping of Quasi-PPOWAD : $R^{n} x R^{n} \rightarrow R$ that has an associated weight vector $\mathrm{W}$ of dimension $n$, where $w_{j} \in[0,1]$ and $\sum_{j=1}^{n} w_{j}=1$, so that

$$
\begin{gathered}
\text { Quasi-PPOWAD }\left(\left\langle x_{1}, y_{1}\right\rangle, \ldots,\left\langle x_{n}, y_{n}\right\rangle\right) \\
=g^{-1} \sum_{i=1}^{q} \sum_{h=1}^{n_{i}} b_{j} \hat{v}_{i j} g\left(C_{i j}(z)\right)
\end{gathered}
$$

where $b_{j}$ is the jth largest of the differences $\left|x_{i}-y_{i}\right|$, $\hat{v}_{i j}$ is the corresponding weight of the $j$ th criteria in the $i$ th category, $i=1, \ldots, q, j=1, \ldots, i_{i}$, and $C_{i j}(z)$ measures the satisfaction of the $j$ th criteria in the $i$ th group by the $\left|x_{i}-y_{i}\right|$ value of each criteria, for each $i=1, \ldots, q, j=1, \ldots, i_{i}$. Additionally, each element has an associated probability $p_{i}$ with $\sum_{i=1}^{n} p_{i}=1$ and $p_{i} \in[0,1], \hat{v}_{j}=\beta w_{j}+$ $(1-\beta) p_{j}$, where $\beta \in[0,1], p_{j}$ is the probability of $p_{i}$ and $g\left(C_{i j}(z)\right)$ is a continuous strictly monotonic function.

Another case is when there is not a probability vector in the formulation, so the PIPOWAD operator becomes the PIOWAD operator. The definition is as follows:

Definition 13. A prioritized induced OWA distance (PIOWAD) operator of dimension $\mathrm{n}$ is a mapping of PIOWAD : $R^{n} x R^{n} \rightarrow R$ that has an associated weight vector $\mathrm{w}$ of dimension $n$, where $w_{j} \in[0,1]$ and $\sum_{j=1}^{n} w_{j}=1$, so that

$$
\begin{aligned}
& \text { PIOWAD }\left(\left\langle u_{1}, x_{1}, y_{1}\right\rangle, \ldots,\left\langle u_{n}, x_{n}, y_{n}\right\rangle\right) \\
& =\sum_{i=1}^{q} \sum_{h=1}^{n_{i}} b_{j} \hat{v}_{i j} g\left(C_{i j}(x)\right)
\end{aligned}
$$

where $b_{j}$ is the jth largest of the differences $\left|x_{i}-y_{i}\right|$, $\left|x_{i}-y_{i}\right|$ is the argument variable represented in the form of individual distances according to $u_{t}, u_{i}$ is the induced order of the variables, $\hat{v}_{i j}$ is the corresponding weight of the $j$ th criteria in the $i$ th category, $i=1, \ldots, q, j=1, \ldots, i_{i}$ and $C_{i j}(x)$ measures the satisfaction of the $j$ th criteria in the $i$ th group by alternative $x \in X$, for each $i=1, \ldots, q, j=1, \ldots, i_{i}$ and $g\left(C_{i j}(z)\right)$ is a continuous strictly monotonic function.

The families of the Quasi-PIPOWAD operator can be separated based on the weighting vector $\mathrm{W}$, the parameter $\lambda$ and the probability vector $p$. The primary families are presented in Table 6 .

\section{Transparency and access to information laws with PIPOWAD operators in Mexico}

\subsection{Theoretical approach}

Transparency can be defined as the openness of the government in informing citizens of how decisions 
are being made, what procedures are used and the consequences of those decisions [1]. In Mexico, the IDAIM measures the quality of the transparency laws in relation to the best national and international practices in the area. This indicator is composed of three main variables: the normative design $\left(v_{1}\right)$, the institutional design $\left(v_{2}\right)$ and the procedures for access to public information and transparency obligations $\left(v_{3}\right)$.

In the specific case of Durango, there is an organization in charge of analyzing and generating new ideas to improve the level of transparency and access to information. It is named the Duranguense Institute of Access to public information and protection of personal data (IDAIP is the acronym in Spanish). To use the PIPOWAD operator to forecast the future ranking of Durango in 2017, based on the information obtained by the directors of the IDAIP, the following steps are followed:

Step 1. We must obtain the expectations of the directors (in this case 3 people) concerning the future valuation for each of the three variables in the IDAIM. This information is based on the changes being implemented to improve Durango's current valuation (5.143 for 2015).

Step 2. The distance of the expectations for 2017 are compared with the results from Coahuila. This is because Coahuila is ranked first (see Annex 1).

Step 3. The distance results for each expert are unified by being multiplied by the prioritized vector. The value for each weight in the prioritized vector is based on the knowledge and experience that each director has in the area.

Step 4. Once the unified results are obtained, they are multiplied by the probabilistic vector and the induced weighted vector. The information for these vectors is obtained based on the decision makers' expertise.

Step 5. Finally, the results for the PIPOWAD are obtained and compared with the other operators (OWAD, PPOWAD and PIOWAD operators).

\subsection{Numerical example}

In this section, we present the results of the investigation of the IDAP of Durango based on the directors' expectations concerning the next IDAIM result. To develop these results, we apply the steps that were defined in Section 5.1.

Step 1. The future expectations concerning Durango according to the directors of the IDAIP are the following (being $e_{1}, e_{2}$, and $e_{3}$ the experts and $v_{1}$, $v_{2}$, and $v_{3}$ the three main variables explained in Section 5.1) (See Table 7)

Step 2. The results for each variable in the case of Coahuila are the following (See Table 8)

With the information in Tables 7 and 8, we obtain the distances between the results. These are as follow (See Table 9).

Step 3. The prioritized vector is $\operatorname{Pr}=(0.4,0.3,0.3)$. This is based on the number of years that the experts have been working at the institution. The unified distance is shown in Table 10 (Being $e_{u}$ the unified opinion of the experts according to the prioritized vector).

Step 4. According to the experts, the probability vector is $P=(0.5,0.3,0.3)$, the weighted vector is $W=(0.4,0.35,0.25)$, the heavy weighting vector $H W=(0.4,0.4,0.3)$ and the induced vector is $U=(5,15,10)$. With this information, we calculate the final difference based on the OWAD, POWAD, IOWAD, PrOWAD, PPOWAD, PIOWAD, PIPOWAD, HOWAD, HPOWAD, HIOWAD, HPrOWAD, HPPOWAD, HPIOWAD and HPIPOWAD operators, as shown in Table 11 (In order to understand how the calculate was done please see Example 1 in Section 3).

With the distance provided by the different operators, we can forecast the score that Durango will have in the IDAIM in 2017. The result is shown in Table 12.

Table 7

Expectations of the experts

\begin{tabular}{cccc}
\hline & $v_{1}$ & $v_{2}$ & $v_{3}$ \\
\hline$e_{1}$ & 9 & 8 & 8 \\
$e_{2}$ & 8.5 & 8 & 9 \\
$e_{3}$ & 9 & 8 & 8 \\
\hline
\end{tabular}

Table 8

Results for Coahuila in 2015

\begin{tabular}{cccc}
\hline & $v_{1}$ & $v_{2}$ & $v_{3}$ \\
\hline Coahuila & 9.8 & 8.3 & 9.8 \\
\hline
\end{tabular}

Table 9

Distance between the expectations of the expert and the best scenario (Coahuila)

\begin{tabular}{cccc}
\hline & $v_{1}$ & $v_{2}$ & $v_{3}$ \\
\hline$e_{1}$ & 0.8 & 0.3 & 1.8 \\
$e_{2}$ & 1.3 & 0.3 & 0.8 \\
$e_{3}$ & 0.8 & 0.3 & 1.8 \\
\hline
\end{tabular}


Table 10

Prioritized distance for Durango

\begin{tabular}{cccc}
\hline & $v_{1}$ & $v_{2}$ & $v_{3}$ \\
\hline$e_{u}$ & 0.98 & 0.30 & 1.45 \\
\hline
\end{tabular}

Table 11

Distance between Durango and Coahuila according to different operators

\begin{tabular}{lccc}
\hline Operators & $v_{1}$ & $v_{2}$ & $v_{3}$ \\
\hline OWAD & 0.3383 & 0.1200 & 0.3667 \\
POWAD & 0.1933 & 0.0900 & 0.4400 \\
IOWAD & 0.1353 & 0.0300 & 0.1283 \\
PrOWAD & 0.3325 & 0.1200 & 0.3750 \\
PPOWAD & 0.1663 & 0.0360 & 0.1125 \\
PIOWAD & 0.1933 & 0.0225 & 0.1540 \\
PIPOWAD & 0.1900 & 0.0225 & 0.1575 \\
HOWAD & 0.2900 & 0.1200 & 0.5867 \\
HPOWAD & 0.1933 & 0.0900 & 0.4400 \\
HIOWAD & 0.1160 & 0.0480 & 0.1760 \\
HPrOWAD & 0.2850 & 0.1200 & 0.6000 \\
HPPOWAD & 0.1425 & 0.0360 & 0.1800 \\
HPIOWAD & 0.1933 & 0.0360 & 0.1320 \\
HPIPOWAD & 0.1900 & 0.0360 & 0.1350 \\
\hline
\end{tabular}

Table 12

Forecast for IDAIM score for Durango in 2017

\begin{tabular}{lccc}
\hline Operators & $v_{1}$ & $v_{2}$ & $v_{3}$ \\
\hline OWAD & 9.4617 & 8.1800 & 9.4333 \\
POWAD & 9.6067 & 8.2100 & 9.3600 \\
IOWAD & 9.6647 & 8.2700 & 9.6717 \\
PrOWAD & 9.4675 & 8.1800 & 9.4250 \\
PPOWAD & 9.6338 & 8.2640 & 9.6875 \\
PIOWAD & 9.6067 & 8.2775 & 9.6460 \\
PIPOWAD & 9.6100 & 8.2775 & 9.6425 \\
HOWAD & 9.5100 & 8.1800 & 9.2133 \\
HPOWAD & 9.6067 & 8.2100 & 9.3600 \\
HIOWAD & 9.6840 & 8.2520 & 9.6240 \\
HPrOWAD & 9.5150 & 8.1800 & 9.2000 \\
HPPOWAD & 9.6575 & 8.2640 & 9.6200 \\
HPIOWAD & 9.6067 & 8.2640 & 9.6680 \\
HPIPOWAD & 9.6100 & 8.2640 & 9.6650 \\
\hline
\end{tabular}

As can be observed, for different operators. the future score for Durango changes because the information that each operator considers is different. It is important to note that the HPIPOWAD operator adds more complexity and provides more information to the decision maker, which is why we consider these results to be more complete than those obtained from the other operators. However, it is also important to analyze the different scenarios that the other operators provide.

In the case of the IDAIM score for Durango in 2017 , it can be observed that it improves dramatically in comparison to the result in 2015 . The experts that work at IDAIP share the opinion that the legislation in 2015 was obsolete, which is why the score of $v_{1}$ for that year was so low. Additionally, they consider that with the new law and different constitutional reforms that provide autonomy to the IDAIP, they can work to improve the valuation of $v_{2}$. Finally, in the case of the score obtained for $v_{3}$, they are creating different campaigns to improve the culture of openness and transparency, as well as to reveal the obligations of the government institutions.

It is important to note that the HPIPOWAD operator is an useful technique that can be used to measure transparency and access to information laws in Mexico, because in the formulas that have been used in the IDAIM and also in the National Institute of Transparency, access to information and protection of personal data (INAI in Spanish acronym), they are applied considering that all the information that is provided is equally important not taking into account who are the decision makers and if they results have to be prioritized, also they don't take into account the probabilities that some of the scenarios can changed or that the results can range out from the maximum or the minimum of the data recollected.

\section{Conclusions}

The main objective of this paper is to present an extension of the hamming distance and the OWA operator. In this sense, the prioritized induced probabilistic ordered weighted average distance (PIPOWAD) and the heavy prioritized induced probabilistic ordered weighted average distance (HPIPOWAD) operators are presented. The main contribution of this operator is that can be used to analyze complex situations where an ideal situation has to be compared with the real one based not only in the distance, but information of the knowledge and expectations of the group decision maker is included by using probabilistic, induced, prioritized and heavy vectors.

In the paper, we present the key definitions and some of the properties of the PIPOWAD operator. Additionally, the families of operators, such as quasiarithmetic, generalized and quadratic, are presented. These families are important because when the problem that we want to solve is not complex or has some specific characteristics, it is possible to use these other formulations.

These new formulations are used in order to measure government transparency based on the IDAIM 
score for Durango, that with the information provided by the group decision making can be seen that there is a high expectation that in the future the results for the state will be closer to the ones that were obtained by Coahuila in 2015. Also, it is possible to analyze different scenarios based on the type of operator that has been used. The basic formulations are important ones when the problem is not that complex but if we want to add more information to the results the complete formulations of the PIPOWAD and HPIPOWAD operator provide a better result.

For future research it is possible to use new methodologies to unify the information provided by the different experts using uncertainty techniques like moving averages [7], linguistic variables [57], the expertons [2] and the forgotten effects methodology [33].

\section{Conflict of interest statement}

All authors declare: "We have no conflict of interest to declare".

\section{References}

[1] A. Florini, The end of secrecy, Foreign Policy (1998), 50-63.

[2] A. Kaufmann, Theory of expertons and fuzzy logic, Fuzzy Sets and Systems 28(3) (1988), 295-304.

[3] A.C. Quinn, Keeping the citizenry informed: Early congressional printing and 21 st century information policy, Government Information Quarterly 20(3) (2003), 281-293.

[4] C. Grønbech-Jensen, The Scandinavian tradition of open government and the European Union: Problems of compatibility? Journal of European Public Policy 5(1) (1998), 185-199.

[5] C. Wei, Z. Pei and H. Li, An induced OWA operator in coal mine safety evaluation, Journal of Computer and System Sciences 78(4) (2012), 997-1005.

[6] E. León-Castro, E. Avilés-Ochoa and J.M. Merigó, Induced heavy moving averages, International Journal of Intelligent Systems (2017). DOI: 10.1002/int.21916

[7] E. León-Castro, E. Avilés-Ochoa and E.A.M. Gil-Lafuente, Exchange rate USD/MXN forecast through econometric models, time series and HOWMA operators, Economic Computation and Economic Cybernetics Studies and Research 50 (2016), 135-150.

[8] E. Vizuete-Luciano, J.M. Merigó, A.M. Gil-Lafuente and S. Boria-Reverter, Decision making in the assignment process by using the Hungarian algorithm with OWA operators, Technological and Economic Development of Economy 21(5) (2015), 684-704.

[9] E.K. Zavadskas and Z. Turskis, Multiple criteria decision making (MCDM) methods in economics: An overview, Technological and Economic Development of Economy 17(2) (2011), 397-427.
[10] F. Blanco-Mesa, J.M. Merigó and J. Kacprzyk, Bonferroni means with distance measures and the adequacy coefficient in entrepreneurial group theory, Knowledge-Based Systems 111 (2016), 217-227.

[11] F.M. Ma, Y.J. Guo and P.T. Yi, Cluster reliability induced OWA operators, International Journal of Intelligent Systems 27(9) (2012), 823-836.

[12] G. Beliakov, A. Pradera and T. Calvo, Aggregation functions: A guide for practitioners, Berlin: Springer-Verlag, 2007.

[13] G. Wei, Hesitant fuzzy prioritized operators and their application to multiple attribute decision making, KnowledgeBased Systems 31 (2012), 176-182.

[14] H. Wang, Y.J. Xu and J.M. Merigó, Prioritized aggregation for non-homogeneous group decision making in water resource management, Economic Computation \& Economic Cybernetics Studies \& Research 48 (2014), 247-258.

[15] J. Fodor, J.L. Marichal and M. Roubens, Characterization of the ordered weighted averaging operators, IEEE Transactions on Fuzzy Systems 3 (1995), 236-240.

[16] J. Gil-Aluja, Elements for a theory of decision in uncertainty (Vol. 32), Springer Science \& Business Media, 1999.

[17] J.A. Shuler, P.T. Jaeger and J.C. Bertot, Implications of harmonizing e-government principles and the Federal Depository Library Program (FDLP), Government Information Quarterly 27(1) (2010), 9-16.

[18] J.C. Bertot, P.T. Jaeger, L.A. Langa and C.R. McClure, Public access computing and Internet access in public libraries: The role of public libraries in e-government and emergency situations, First Monday 11(9) (2006).

[19] J.M. Merigó and A.M. Gil-Lafuente, Decision-making in sport management based on the OWA operator, Expert Systems with Applications 38(8) (2011), 10408-10413.

[20] J.M. Merigó and A.M. Gil-Lafuente, New decisionmaking techniques and their application in the selection of financial products, Information Sciences 180(11) (2010), 2085-2094.

[21] J.M. Merigó and A.M. Gil-Lafuente, The induced generalized OWA operator, Information Sciences 179(6) (2009), 729-741.

[22] J.M. Merigó and A.M. Gil-Lafuente, The ordered weighted averaging distance operator, Lectures on Modelling and Simulation 8(1) (2007), 1-11.

[23] J.M. Merigó and M. Casanovas, A new Minkowski distance based on induced aggregation operators, International Journal of Computational Intelligence Systems 4(2) (2011a), 123-133.

[24] J.M. Merigó and M. Casanovas, Decision-making with distance measures and induced aggregation operators, Computers \& Industrial Engineering 60(1) (2011b), 66-76.

[25] J.M. Merigó and M. Casanovas, Induced and heavy aggregation operators with distance measures, Journal of Systems Engineering and Electronics 21(3) (2010), 431-439.

[26] J.M. Merigó, D. Palacios-Marqués and P. Soto-Acosta, Distance measures, weighted averages, OWA operators and Bonferroni means, Applied Soft Computing 50 (2017), 356-366.

[27] J.M. Merigó, Decision-making under risk and uncertainty and its application in strategic management, Journal of Business Economics and Management 16(1) (2015), 93-116.

[28] J.M. Merigó, M. Casanovas and D. Palacios-Marqués, Linguistic group decision making with induced aggregation operators and probabilistic information, Applied Soft Computing 24 (2014), 669-678. 
[29] J.M. Merigó, M. Casanovas and S. Zeng, Distance measures with heavy aggregation operators, Applied Mathematical Modelling 38(13) (2014), 3142-3153.

[30] J.M. Merigó, Probabilities in the OWA operator, Expert Systems with Applications 39 (2012), 11456-11467.

[31] J.M. Merigó, Y. Xu and S. Zeng, Group decision making with distance measures and probabilistic information, Knowledge-Based Systems 40 (2013), 81-87.

[32] J.Y. Dong and S.P. Wan, A new method for prioritized multicriteria group decision making with triangular intuitionistic fuzzy numbers, Journal of Intelligent \& Fuzzy Systems 30(3) (2016), 1719-1733.

[33] Kaufmann and J. Gil-Aluja, Models for the research of forgotten effects. (In Spanish), Spain: Milladoiro, 1988.

[34] L.A. Pérez-Arellano, E. León-Castro, E. Avilés-Ochoa and J.M. Merigó, Prioritized induced probabilistic operator and its application in group decision making, International Journal of Machine Learning and Cybernetics (2017). DOI: 10.1007/s13042-017-0724-2

[35] M. Agarwal, M. Hanmandlu and K.K. Biswas, A probabilistic and decision attitude aggregation operator for intuitionistic fuzzy environment, International Journal of Intelligent Systems 28(8) (2013), 806-839.

[36] P. Liu, F. Jin, X. Zhang, Y. Su and M. Wang, Research on the multi-attribute decision-making under risk with interval probability based on prospect theory and the uncertain linguistic variables, Knowledge-Based Systems 24(4) (2011), 554-561.

[37] P. Liu and Y. Wang, Interval neutrosophic prioritized OWA operator and its application to multiple attribute decision making, Journal of Systems Science and Complexity 29(3) (2016), 681-697.

[38] R.R. Yager and D.P. Filev, Induced ordered weighted averaging operators, IEEE Transactions on Systems, Man and Cybernetics (1999), 141-150.

[39] R.R. Yager, Generalized OWA aggregation operators, Fuzzy Optimization and Decision Making 3(1) (2004), 93-107.

[40] R.R. Yager, Heavy OWA operators, Fuzzy Optimization and Decision Making 1(4) (2002), 379-397.

[41] R.R. Yager, Modeling prioritized multicriteria decision making, IEEE Transactions on Systems, Man and Cybernetics 34 (2004), 2396-2404.

[42] R.R. Yager, On ordered weighted averaging aggregation operators in multicriteria decisionmaking, IEEE Transactions on Systems, Man and Cybernetics 18(1) (1988), 183-190.

[43] R.R. Yager, Prioritized aggregation operators, International Journal of Approximate Reasoning 48 (2008), 263-274.

[44] R.R. Yager, Prioritized OWA aggregation, Fuzzy Optimization and Decision Making 8 (2009), 245-262.
[45] R.W. Hamming, Error detecting and error correcting codes, Bell Labs Technical Journal 29(2) (1950), 147-160.

[46] S. Chen, Z. Mu and S. Zeng, Atanassov's intuitionistic fuzzy decision making with probabilistic information and distance measure, Journal of Intelligent \& Fuzzy Systems 28(1) (2015), 317-325.

[47] S. Greco, J. Figueira and M. Ehrgott, Multiple criteria decision analysis, Springer's International Series, 2005.

[48] S. Wan, J. Dong and D. Yang, Trapezoidal intuitionistic fuzzy prioritized aggregation operators and application to multi-attribute decision making, Iranian Journal of Fuzzy Systems 12(4) (2015), 1-32.

[49] S. Zeng and W. Su, Intuitionistic fuzzy ordered weighted distance operator, Knowledge-Based Systems 24(8) (2011), $1224-1232$

[50] S. Zeng, J.M. Merigo and W. Su, The uncertain probabilistic OWA distance operator and its application in group decision making, Applied Mathematical Modelling 37(9) (2013), 6266-6275.

[51] S. Zeng, J.M. Merigó, D. Palacios-Marqués, H. Jin and F. $\mathrm{Gu}$, Intuitionistic fuzzy induced ordered weighted averaging distance operator and its application to decision making, Journal of Intelligent \& Fuzzy Systems 32(1) (2017), 11-22.

[52] S. Zeng, W. Su and C. Zhang, Intuitionistic fuzzy generalized probabilistic ordered weighted averaging operator and its application to group decision making, Technological and Economic Development of Economy 22(2) (2016), 177-193.

[53] S.Z. Zeng, An extension of OWAD operator and its application to uncertain multiple-attribute group decision-making, Cybernetics and Systems 47(5) (2016), 363-375.

[54] T. Calvo, G. Mayor and R. Mesiar, Aggregation operators: New trends and applications, Physica-Verlag, New York, 2002.

[55] Y.J. Xu, T. Sun and D.F. Li, Intuitionistic fuzzy prioritized OWA operator and its application in multi-criteria decision-making problem, Control and Decision 26(1) (2011), 129-132.

[56] Z. Shouzhen and C. Su, Extended VIKOR method based on induced aggregation operators for intuitionistic fuzzy financial decision making, Economic Computation \& Economic Cybernetics Studies \& Research 49(4) (2015).

[57] Z. Xu and R.R. Yager, Some geometric aggregation operators based on intuitionistic fuzzy sets, International Journal of General Systems 35(4) (2006), 417-433.

[58] Z. Xu, Induced uncertain linguistic OWA operators applied to group decision making, Information Fusion 7(2) (2006), 231-238.

[59] Z.S. Xu and J. Chen, Ordered weighted distance measures, Journal of Systems Science and Systems Engineering 17(4) (2008), 432-445. 


\section{Annex}

Annex 1

Ranking of the states in Mexico according to IDAIM 2015

\begin{tabular}{|c|c|c|c|c|c|}
\hline Rank & State & $v^{1}$ & $v^{2}$ & $v^{3}$ & Total \\
\hline 1 & Coahuila & 9.8 & 8.3 & 9.8 & 9.339 \\
\hline 2 & Distrito Federal & 8.0 & 8.1 & 8.1 & 8.111 \\
\hline 3 & Colima & 8.0 & 7.1 & 7.8 & 7.713 \\
\hline 4 & Puebla & 6.0 & 7.3 & 8.0 & 7.130 \\
\hline 5 & Guerrero & 6.1 & 7.1 & 7.2 & 6.870 \\
\hline 6 & Nayarit & 6.8 & 6.2 & 7.1 & 6.726 \\
\hline 7 & Chihuahua & 7.1 & 5.8 & 7.1 & 6.706 \\
\hline 8 & Jalisco & 7.3 & 6.4 & 6.1 & 6.614 \\
\hline 9 & Morelos & 6.6 & 6.4 & 6.6 & 6.569 \\
\hline 10 & Oaxaca & 7.1 & 6.0 & 6.3 & 6.519 \\
\hline 11 & Nuevo Leon & 6.9 & 6.0 & 6.2 & 6.424 \\
\hline 12 & Zacatecas & 7.4 & 5.4 & 6.2 & 6.394 \\
\hline 13 & Tlaxcala & 6.6 & 6.2 & 6.2 & 6.381 \\
\hline 14 & Veracruz & 6.0 & 5.0 & 7.3 & 6.167 \\
\hline 15 & Durango & 6.3 & 5.4 & 6.6 & 6.149 \\
\hline 16 & Michoacán & 7.3 & 5.6 & 5.3 & 6.112 \\
\hline 17 & Baja California & 5.2 & 6.2 & 6.5 & 5.988 \\
\hline 18 & San Luis Potosi & 5.3 & 5.8 & 6.6 & 5.957 \\
\hline 19 & Aguascalientes & 6.1 & 4.9 & 5.8 & 5.657 \\
\hline 20 & Sonora & 5.5 & 5.0 & 6.2 & 5.633 \\
\hline 21 & Tabasco & 6.1 & 4.7 & 5.8 & 5.594 \\
\hline 22 & Chiapas & 5.8 & 5.8 & 4.6 & 5.449 \\
\hline 23 & Quintana Roo & 5.2 & 5.2 & 5.1 & 5.215 \\
\hline 24 & Estado de Mexico & 4.6 & 5.6 & 5.2 & 5.171 \\
\hline 25 & Sinaloa & 6.0 & 3.7 & 5.6 & 5.143 \\
\hline 26 & Tamaulipas & 5.3 & 4.5 & 5.5 & 5.142 \\
\hline 27 & Guanajuato & 5.5 & 4.5 & 4.8 & 4.986 \\
\hline 28 & Campeche & 5.7 & 4.3 & 4.8 & 4.976 \\
\hline 29 & Querétaro & 4.7 & 4.3 & 5.7 & 4.950 \\
\hline 30 & Yucatan & 4.7 & 4.1 & 5.8 & 4.929 \\
\hline 31 & Baja California Sur & 4.7 & 3.9 & 5.5 & 4.741 \\
\hline 32 & Hidalgo & 4.1 & 4.9 & 4.7 & 4.594 \\
\hline
\end{tabular}

